\title{
6G Mobile Communication Networks: Key Services and Enabling Technologies
}

\author{
Sanjay Kumar \\ Birla Institute of Technology, Mesra, India \\ E-mail: skumar@bitmesra.ac.in \\ Received 06 July 2021; Accepted 05 December 2021; \\ Publication 11 February 2022

\begin{abstract}
The deployment of 5G (fifth generation mobile communication) network has already started. Although, 5G will offer significant improvements over the existing systems, but will not be able to fulfil the emerging future demands. Therefore, development of communication networks beyond $5 \mathrm{G}$ will be required, leading to $6 \mathrm{G}$. $6 \mathrm{G}$ will satisfy unprecedented requirements and expectations that $5 \mathrm{G}$ cannot meet. This paper highlights various new services to be offered by $6 \mathrm{G}$, and a few enabling technologies for $6 \mathrm{G}$ development.
\end{abstract}

Keywords: 5G, 6G, mobile hologram, machine communication, artificial intelligence.

\section{Introduction}

International Telecommunication Union-Radiocommunication Sector (ITUR) envisioned IMT-2020 which represents 5G. ITU-R will probably release IMT-2030, which will summarize the possible requirements of mobile communications in 2030. This will represent 6G. Deployment of 5G has already been started worldwide. 3 GPP release 17 focuses on further enhancements

Journal of ICT Standardization, Vol. 10_1, 1-10.

doi: 10.13052/jicts2245-800X.1011

(C) 2022 River Publishers 


\section{S. Kumar}

to the $5 \mathrm{G}$ system and enablers for new features and services. Although, $5 \mathrm{G}$ will offer significant improvements over the existing systems, but will not be able to fulfil the emerging future demands [1]. There will be need for more advanced services by 2030 [2]. In fact, wireless communications application is expanding from connecting humans to connecting things. $6 \mathrm{G}$ will satisfy unprecedented requirements and expectations that 5G cannot meet [3]. 6G will provide ultimate experience for all through hyper-connectivity involving humans and everything [4]. 6G will have primarily the same Key Performance Indicators (KPIs) as 5G, but with significantly enhanced performance targets. Table 1 gives a comparative overview of $5 \mathrm{G}$ and $6 \mathrm{G}$ in terms of various KPIs.

It is unlikely that all of the above mentioned KPIs will be supported simultaneously. Different use cases will require different sets of KPIs. Due to extremely high data rate and low-latency communication, $6 \mathrm{G}$ can support various applications such as telemedicine, distance education, innovative entertainment services and autonomous operation of various equipment including cars. Other autonomous mobility solutions like drone delivery systems and drone swarms are also evolving in different application areas such as military, emergency response and construction. 6G will support intelligent networks, heterogeneous networks, convergence, distributed caching, sensing, IoT applications and artificial intelligence-based decisions. In fact, 6G will provide hyper connectivity among people and everything and will eliminate the constraints of place and time. The $6 \mathrm{G}$ system will provide full Internet of everything ( $\mathrm{IoE}$ ) support. IoE is an umbrella term that introduces seamless integration and autonomous coordination among a very large number of computing elements and sensors, physical objects or devices, people,

Table 1 Comparative overview of 5G and 6G in terms of various KPIs

\begin{tabular}{lcc}
\hline KPIs & $5 \mathrm{G}$ & $6 \mathrm{G}$ \\
\hline Peak Data Rate & $20 \mathrm{~Gb} / \mathrm{s}$ & $1 \mathrm{~Tb} / \mathrm{s}$ \\
User-experienced Data Rate & $0.1 \mathrm{~Gb} / \mathrm{s}$ & $1 \mathrm{~Gb} / \mathrm{s}$ \\
Peak Spectral Efficiency & $30 \mathrm{~b} / \mathrm{s} / \mathrm{Hz}$ & $60 \mathrm{~b} / \mathrm{s} / \mathrm{Hz}$ \\
User-experienced Spectral Efficiency & $0.3 \mathrm{~b} / \mathrm{s} / \mathrm{Hz}$ & $3 \mathrm{~b} / \mathrm{s} / \mathrm{Hz}$ \\
Maximum Bandwidth & $1 \mathrm{GHz}$ & $100 \mathrm{GHz}$ \\
Connection Density & $10^{6}$ devices $/ \mathrm{km}^{2}$ & $10^{7}$ devices $/ \mathrm{km}^{2}$ \\
Latency & $1 \mathrm{~ms}$ & $0.1 \mathrm{~ms}$ \\
Jitter & not specified & $1 \mu \mathrm{s}$ \\
Mobility & $500 \mathrm{~km} / \mathrm{h}$ & $1000 \mathrm{~km} / \mathrm{h}$ \\
\hline
\end{tabular}


processes, and data into one system using the internet infrastructure. IoE will be used for smart societies, such as smart cars, smart health, and smart industries.

\section{New Use Cases and Services Offered by 6G}

$6 \mathrm{G}$ will continue to enhance the use cases of $5 \mathrm{G}$ services, i.e., Enhanced Mobile Broad Band (eMBB), Ultra Reliable and Low Latency Communications (URLLC), and Massive Machine Type Communications (mMTC). In addition, there will be several new services such as Immersive XR (Extended Reality), Mobile Hologram, Virtual Replica, Machine Communication, Haptic Communication and Communication as Social Infrastructure. These are briefly explained below.

- XR Experience

- Mobile Hologram

- Virtual Replica

- Machine Communication

- Haptic Communication

- Communication as Social Infrastructure

\subsection{XR Experience}

XR represents combination of VR (Virtual Reality), AR (Augmented Reality), and MR (Mixed Reality). XR is based on capturing multi-sensory inputs and providing real-time user interactions remotely. 5G will not be able to keep pace with seamless streaming of XR experience [4]. Extremely high-data-rate, low latency, and highly reliable wireless connectivity provided by $6 \mathrm{G}$ will help deliver a fully immersive XR experience. XR offers new possibilities in various fields such as entertainment, medicine, education, and manufacturing. XR experience requires extremely high computing capabilities and data rate performance.

\subsection{Mobile Hologram}

Hologram is a next-generation media technology that can present gestures and facial expressions by means of a holographic display, where the content for display can be obtained by means of real-time capture, transmission, and 3D rendering techniques [4]. Providing hologram display of real-time services requires extremely high data rate transmission. Moreover, support 
of a human-sized hologram requires significantly much higher data rate. With a peak data rate of $20 \mathrm{Gbps}, 5 \mathrm{G}$ cannot support hologram display in real-time. Artificial Intelligence in $6 \mathrm{G}$ can be leveraged to achieve efficient compression, extraction, and rendering of the hologram display. Holographic telepresence will help build smart healthcare system.

\subsection{Virtual Replica}

In future, physical entities such as people, devices, objects, systems, and even places are expected to be replicated in virtual world [4]. This virtual replica of a physical entity is called a digital twin. In fact, digital twin is a representation of a remotely controlled set of sensors and actuators. Users' interaction with digital twin can result in actions in the physical world [4]. Users will be able to observe changes or detect problems remotely through the representations offered by digital twins. In $6 \mathrm{G}$, users will be able to explore and monitor the reality in a virtual world without temporal or spatial constraints using digital twins.

\subsection{Machine Communication}

Machines will be the dominant users of wireless communication in future. The number of connected machines is growing exponentially. Examples of connected machines include home appliances, office accessories, factory equipment, sensors, robots, vehicles, drones etc. Historically wireless communication services have been developed for humans. In 5G, machines are also considered in defining requirements and developing technologies. However, 6G will have to be developed specifically to connect extremely large number of machines, for which much higher performance targets have to be set. Table 2 gives a comparative overview of the perception capabilities of humans and machines, which may give an insight on the performance targets needed for machine communication.

Table 2 A comparative overview of the perception capabilities of humans and machines [4]

\begin{tabular}{|l|c|l|}
\hline Perception Capability & Humans & \multirow{2}{*}{ Machines } \\
\cline { 1 - 2 } Maximum Resolution & $1 / 150^{\circ}$ & \multirow{2}{*}{$\begin{array}{l}\text { Exceeds human } \\
\text { limitations! }\end{array}$} \\
\hline Viewing Angle & Azimuth $200^{\circ}$, Elevation $130^{\circ}$ & \\
\hline Visible Wavelength & $280-780 \mathrm{~nm}$ & \\
\hline Latency Perception & Less than $100 \mathrm{~ms}$ & \\
\hline Audible Frequency & $250-2,000 \mathrm{~Hz}$ & \\
\hline
\end{tabular}


Perception capabilities of machines exceed human limitations. For example, machine vision is not constrained since it can take advantage of many cameras with various functions. $6 \mathrm{G}$ requires much higher performance targets due to high machines communication.

\subsection{Haptic Communication}

Human being uses five senses of hearing, sight, taste, smell, and touch to experience the world around. 6G aims to remotely transfer data obtained from the five senses. This calls for using neurological process through sensory integration by detecting the sensations from the human body and the environment. Haptic communication is a branch of nonverbal communication that uses the sense of touch. The proposed $6 \mathrm{G}$ wireless communication will support haptic communication, where the remote users will be able to enjoy haptic experiences through real-time interactive systems.

\subsection{Communication as Social Infrastructure}

Wireless communication has become an integral part of social infrastructure and is expected to play a pivotal role in solving various social issues such as climate change, poverty and education inequality. United Nations has set Sustainable Development Goals (SDGs) which aims to solve various social issues, eradicate poverty and achieve sustainable development by 2030 world-wide. SDG is like a blueprint to achieve a better and more sustainable future for all. Realizing SDG will require intensive application of Information and Communication Technologies (ICT), where $6 \mathrm{G}$ will play very important role to enable access to required information, resources and social services without constraints of time and physical location.

\section{Key Enabling Technologies for 6G}

6G has more diversified and advance requirements. The technological requirements for $6 \mathrm{G}$ implementation will be based on enhancement of existing technologies, and the new technologies that were not considered while developing 5G. A few key enabling technologies for $6 \mathrm{G}$ are enlisted and discussed below.

- Artificial Intelligence based Communication

- Software Centric Networks

- Terahertz Communication 
- Ultra-massive MIMO

- New Network Topology

- Intelligent Reflecting Surface

\subsection{Artificial Intelligence Based Communication}

Application of Artificial Intelligence (AI) in communication networks can improve performance in many aspects. There is a limit to what is achievable today as AI is not used significantly in communication networks. Today AI technologies are becoming available for practical applications, hence AI based communication networks can be developed. AI can be used for learning, reasoning, detection, analysis, prediction, decision making and network planning. AI can learn the environment and adopt appropriate solutions which enables equipment to make independent decisions. This results into "Intelligent connectivity", which is one of the prime features of 6G [3]. The current support of AI for $5 \mathrm{G}$ can only be regarded as the optimization of traditional network architecture, rather than a new intelligent communication network [3]. AI will be fully integrated with 6G. In fact, building $6 \mathrm{G}$ network based on AI will be inevitable. Currently AI technologies are becoming available for practical applications, hence it is becoming feasible to develop AI based communication networks. AI helps to reproduce the real world in cyberspace and emulates it so that future predictions can be possible. Currently there is an increasing trend towards cyber-physical fusion, which is becoming a reality due to feasibility of implementation of artificial intelligence.

\subsection{Software Centric Network}

There is increasing trend towards virtualization of network functions. Network Function Virtualization (NFV) enables separation of hardware from software (or functions). This enables network infrastructure to be decoupled from the services provided by the network. This helps to maximizes network utilization by allowing different services to coexist on the same infrastructure. Therefore, different Service Providers (SPs) can dynamically share the same physical network provided by Mobile Network Operator (MNOs). Virtualization of network functions requires shifting to Software Defined Networks (SDN), which performs dynamic reconfiguration of the network topology in order to adjust to load and demand, and enables dynamic radio resource management in a distributed fashion. If required, it directs additional network resources to maintain the Quality of users' Experience (QoE). 
NFV and SDN provide the network operators and service providers with several benefits such as a more manageable means to monitor the network, better support of new feature roll outs and network relocation. This significantly reduces Capital Expenses (CapEx) and Operational Expenses (OpEx) of the network operators and service providers. OpEx is also reduced by power savings, as the network capacity is used only when and where needed. Hence cheaper and simpler network architecture is offered, which can be easily upgraded, and integrated with newer applications. NFV and SDN will be important features of $6 \mathrm{G}$, which will ensure flexibility, reconfigurability and programmability of the networks.

\subsection{Terahertz Communication}

Higher spectrum will be required to meet the extremely high data rate requirements of $6 \mathrm{G}$. In this regard, the Terahertz $(\mathrm{THz})$ band will play an important role in $6 \mathrm{G}$ to support extremely high data rate communication $[5,6]$. $\mathrm{THz}$ waves usually refer to the frequency band between $0.1 \mathrm{THz}$ to $10 \mathrm{THz}$ with the corresponding wavelengths between $0.03 \mathrm{~mm}$ to $3 \mathrm{~mm}$ range [4]. THz band will provide widely available bandwidth to support very high data rates but will results into high path loss, therefore highly directional antennas will most probably be indispensable [3]. Highly directional antennas create narrow beamwidths, which lowers the amount of interference. The small wavelength in the $\mathrm{THz}$ band allows larger number of antenna elements to be incorporated, which support using advanced adaptive array technologies that can overcome the range limitations.

\subsection{Ultra-Massive MIMO}

MIMO exploits spatial domain and improves wireless communication system performance. $5 \mathrm{G}$ considered massive MIMO to maximize beam-forming gain, minimize interference, improve reliability, and to provide robustness to signal transmission. $6 \mathrm{G}$ requires an ultra-high spatial resolution, where each transmitted signal can be focused in a small region around the receiver, leading to a very high beamforming gain. Beamforming gain describes the enhancement in received signal quality because of the adjustment in the antenna array. A further enhancement in massive MIMO is considered for 6G with a greater number of antenna elements, more layers for spatial multiplexing, and a distributed antenna arrangement. This is termed as ultramassive MIMO where the antenna characteristics are varied at a very high 
speed periodically to generate a large number of virtual antennas, and to increase the number of layers for spatial multiplexing.

\subsection{New Network Topology}

$6 \mathrm{G}$ will operate over heterogeneous networks, where users can move seamlessly from one network to another network and the best network will be automatically selected from the available networks. This will eliminate the concept of cells. In cellular structure large variation in throughput performance is observed between cell center and cell edge users. The cell edge users experience low throughput performance. Moreover, in cellular structure users' movement from one cell to another cell causes too many handovers in dense networks, handover failures, handover delays, pingpong handover effect and loss of data. 6G aims to achieve nearly uniform throughput performance regardless of users' position. This could be realized by abandoning the concept of cells. Since the cellular structure minimizes the level of interference in the network. Therefore, abandoning the concept of cells may not be considered good conventionally, since such topology may result into high amount of interference. $6 \mathrm{G}$ aims to avoid such interference by beam control and path selection mechanisms. In 6G the cell-free communication will be realized through multi-connectivity and multi-tier hybrid techniques. Integration with communication through satellites can also create space-based cell free network.

\subsection{Intelligent Reflecting Surfaces}

6G will use intelligent reflecting surface (IRS) to improve the propagation conditions. IRS introduces additional scattering and controls scattering characteristics to create beams towards the intended receivers in order to increase beamforming gains and decrease co-channel interference. Ideally, an IRS can create a smart, programmable, and controllable wireless propagation environment. IRS improves the propagation conditions particularly over short range and very high frequencies (such as near Terahertz frequencies), where relaying is not feasible. IRS can support propagation even when the line-ofsight (LOS) path is blocked. IRS is reconfigurable in real-time so it can adapt to channel variations. IRS can be used for cognitive radio, wireless power transfer, physical layer security, and backscattering, where IoT devices near the surface can communicate with the access point with minimum energy.

Several other techniques will be needed towards 6G development, which includes Optical Wireless Communication (OWC), Integrated access 
and backhaul support, Integration of sensors and communication networks, Advanced interference management schemes, Advanced multiple access techniques, Rate-splitting, In-band full-duplex technology, Machinelearning-based optimization, Improved coding, modulation and waveforms design.

\section{Summary}

$5 \mathrm{G}$ will offer significant improvements over the existing systems, but will not be able to fulfil the emerging future demands. There will be need for more advanced services by 2030 leading to emergence of $6 \mathrm{G}$. This paper discussed several key services offered by $6 \mathrm{G}$ such as XR experience, mobile hologram, virtual replica, machine communication and communication as social infrastructure. $6 \mathrm{G}$ will be based on enhancement of existing technologies, and the new technologies that were not considered while developing 5G. A few key enabling technologies for $6 \mathrm{G}$ will be Artificial Intelligence based Communication, Software Centric Networks, Terahertz Communication, Ultra-massive MIMO, New Network Topology and Intelligent Reflecting Surface, which have been discussed briefly in the paper.

\section{References}

[1] ITU-R M.2370-0, IMT traffic estimates for the years 2020 to 2030, Jul,2015. (available at https://www.itu.int/pub/r-rep-m.2370)

[2] White Paper 5G Evolution and 6G NTT DOCOMO, INC. Jan, 2020. (available at https://www.nttdocomo.co.jp)

[3] Yajun Zhao, Guanghui Yu, Hanqing Xu, "6G Mobile Communication Network: Vision, Challenges and Key Technologies", Scientia Sinica Informationis, 2019.

[4] "6G: The next hyper connected experience for all", Samsung 6G Vision. https://cdn.codeground.org/nsr/downloads/researchareas/6G\%20Vision .pdf

[5] Nandana Rajatheva et al., "White Paper on Broadband Connectivity in 6G”, Electrical Engineering and Systems Science, Signal Processing, 2020. (available at https://arxiv.org/abs/2004.14247)

[6] O. Tervo, t. Levanen, k. Pajukoski, j. Hulkkonen, p. Wainio, and m. Valkama, "5G new radio evolution towards sub-thz communications," in proc. $6 \mathrm{G}$ wireless summit, Mar, 2020. 
10 S. Kumar

\section{Biography}

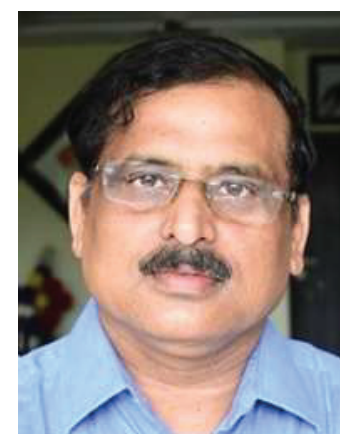

Sanjay Kumar received MBA from Pune University, Pune, India in 1994, M. Tech. in Electronics and Communication Engineering from Guru Nanak Dev Engineering College, Ludhiana, India in 2000, and $\mathrm{PhD}$ in Wireless Communication from Aalborg University, Denmark in 2009. He is an Associate Professor in the department of Electronics and Communications Engineering at Birla Institute of Technology Mesra, India. He served Indian Air Force from 1985 to 2000 in various technical capacities. His research interests lie in the field of Wireless Communication Technology with focus on wireless signal propagation, fading, channel impairment removal techniques, flexible spectrum usage, cognitive radio networks and technologies for $5 \mathrm{G}$ and beyond. 